\title{
Primer registro de cráneos asignados a Palaeospheniscus (Aves, Spheniscidae) procedentes de la Formación Bahía Inglesa (Mioceno Medio-Tardío), Chile
}

\author{
First record of skulls assigned to Palaeospheniscus (Aves, Spheniscidae) from the Bahía \\ Inglesa Formation (Middle-Late Miocene), Chile
}

CAROLINA ACOSTA HOSPITALECHE ${ }^{1} \&$ JHOANN CANTO $^{2}$

\author{
${ }^{1}$ División Paleontología Vertebrados, Museo de La Plata, Paseo del Bosque, 1900 La Plata, Argentina; \\ e-mail: acostacaro@museo.fcnym.unlp.edu.ar \\ ${ }^{2}$ Sección Paleontología, Museo Nacional de Historia Natural, Casilla 787, \\ Santiago, Chile; e-mail: jcanto@paleontologica.org
}

\begin{abstract}
RESUMEN
Los cráneos de los Spheniscidae (como los de todas las aves), aunque escasos en el registro paleontológico, muestran importantes caracteres a nivel taxonómico. Hasta el momento se conocían solo seis cráneos en todo el mundo: Paraptenodytes antarctica y una nueva especie de Spheniscidae procedentes de las Formaciones Gaiman (Mioceno Temprano) y Puerto Madryn (Mioceno Tardío) de Argentina, respectivamente; Spheniscus megaramphus y S. urbinai de la Formación Pisco (Mioceno Tardío) de Perú, Marplesornis novaezealandiae del Plioceno de Nueva Zelanda y un resto de Sphenicidae ef Spheniscus procedente de la Formación Bahía Inglesa (Mioceno Medio-Tardío). De esta última unidad provienen cinco restos craneanos recientemente descubiertos en la localidad de Caldera (Región de Atacama, Chile), los cuales fueron descritos y asignados preliminarmente a Palaeospheniscus. Estos materiales constituyen el primer registro de cráneos para la especie y significan un importante aporte al conocimiento de la avifauna fósil de Chile y en particular de la Formación Bahía Inglesa.
\end{abstract}

Palabras clave: Spheniscidae, Palaeospheniscus, Mioceno Medio-Tardío, Formación Bahía Inglesa, Chile.

\begin{abstract}
Although the skulls of the Spheniscidae are unusual in the paleontologic record, they show important features useful in the systematics of the group, as well as in the rest of the birds. Until now, fossil occurrences were restricted to six skulls: Paraptenodytes antarctica and one new species from the Gaiman Formation (Early Miocene) and Puerto Madryn Formation (Late Miocene) of Argentina, respectively; Spheniscus megaramphus and S. urbinai from the Pisco Formation (Late Miocene), Perú; Marplesornis novaezealandiae from New Zealand (Pliocene); and Spheniscidae cf. Spheniscus from the Bahía Inglesa Formation (Middle-Late Miocene), Chile. Five new materials have been exhumed from this last unit. These skulls, which come from the Caldera locality (Region of Atacama, Chile), have been preliminarily assigned to Palaeospheniscus (Aves, Spheniscidae), constituting the first skulls described for this species. We made an important contribution to the knowledge of the fossil avifauna of Chile and particularly of the Bahía Inglesa Formation.
\end{abstract}

Key words: Spheniscidae, Palaeospheniscus, Middle-Late Miocene, Bahía Inglesa Formation, Chile.

\section{INTRODUCCIÓN}

Los Sphenisciformes constituyen un Orden de aves en el cual la totalidad de las especies son acuáticas marinas y presentan fuertes adaptaciones al buceo. Sus primeros restos fósiles corresponden al Paleoceno Tardío de Isla Seymour, Antártida ${ }^{1}$ (Tambussi et al. en prensa) y se encuentran ampliamente distribuidos en los sedimentos terciarios del Hemisferio Sur, presentando una alta diversidad y abundancia.

La totalidad de las especies actuales se encuentran reunidas en la subfamilia Spheniscinae,

1 TAMBUSSI CP, MA REGUERO, S MARENSSI \& S SANTILLANA (2002) The earliest known penguin and the evolution of spheniscid size. Actas del Primer Congreso Latinoamericano de Paleontología de Vertebrados, Santiago, Chile: Resumen 53. 
mientras que los fósiles se clasifican en cuatro subfamilias según Simpson (1946) o en tres según Marples (1952), quien incluye a los Anthropornithinae en los Palaeeudyptinae. Los Paraptenodytinae, conocidos para el Eoceno Tardío-Oligoceno Temprano de Patagonia, Mioceno Temprano y Mioceno Tardío Temprano de la Patagonia, Argentina (Tonni 1980, Acosta Hospitaleche 2003, 2004), y el Mioceno TardíoPlioceno Temprano de Chile; los Palaeospheniscinae, conocidos para el Mioceno Temprano de Patagonia (Tonni 1980, Acosta Hospitaleche 2004), el Mioceno Tardío-Plioceno Temprano de Chile $^{2}$ y el Mioceno Medio de Perú (Acosta Hospitaleche \& Stucchi 2005); los Anthropornithinae citados para Nueva Zelanda y Antártida (Simpson 1946, Ando 2003); y finalmente los Palaeeudyptinae, representados en el Mioceno de Australia (Simpson 1970) y Eoceno de Antártida (Tambussi et al. en prensa) y Nueva Zelanda (Fordyce \& Jones 1990).

El carácter fragmentario del registro paleontológico ha provocado que la diagnosis de estas Subfamilias, así como la de la mayoría de las especies extintas se haya basado en los caracteres presentes en el húmero y en el tarsometatarso (Simpson 1946). Los cráneos de los Spheniscidae (como los de todas las aves), aunque escasos en el registro paleontológico, exhiben importantes caracteres a nivel taxonómico ${ }^{3}$. Por este motivo, la sistemática de las formas extintas se ha basado en los húmeros y los tarsometatarsos. Hasta el momento solo se conocían cráneos fósiles asignados a Paraptenodytes antarctica Moreno y Mercerat, 1891 (Simpson 1946) y Spheniscidae nov. gen y sp procedentes de las Formaciones Gaiman del Mioceno Temprano y Puerto Madryn del Mioceno Tardío ${ }^{4}$, respectivamente; Spheniscus

\footnotetext{
2 ACOSTA HOSPITALECHE C, O FRITIS, C TAMBUSSI \& A QUINZIO (2002) Nuevos restos de pingüinos (Aves: Spheniscidae) en la Formación Bahía Inglesa (Mioceno superior - Plioceno inferior) de Chile. Actas del Primer Congreso Latinoamericano de Paleontología de Vertebrados, Santiago, Chile Resumen 16. 3 ACOSTA HOPITALECHE C \& G GASPARINI (2004) Análisis multivariado de caracteres cuantitativos craneanos en Spheniscidae (Aves, Sphenisciformes). Reunión de Comunicaciones de la Asociación Argentina de Paleontología, Diamante, 2004. Ameghiniana 41: 4R.

4 COZZUOL MA, C TAMBUSSI \& J NORIEGA (1993) Un pingüino (Aves: Spheniscidae) de la Formación Puerto Madryn (Mioceno Medio) en Península Valdés, Chubut, Argentina, con importantes implicancias filogenéticas. Décimas Jornadas Argentinas de Paleontología de Vertebrados. La Plata, Ameghiniana 30: 327-328.
}

megaramphus Stucchi, Urbina y Giraldo, 2003 (Stucchi et al. 2003) y S. urbinai Stucchi, 2002 de la Formación Pisco del Mioceno Tardío (Stucchi 2002); Marplesornis novaezealandiae (Marples, 1960) del Plioceno de Nueva Zelanda (Marples 1960) y un resto de Sphenicidae cf. Spheniscus Brisson, 1760 procedente de la Formación Bahía Inglesa del Mioceno TardíoPlioceno Temprano (Walsh \& Hume 2001).

Se dan a conocer en este trabajo cinco nuevos restos craneanos procedentes de la Formación Bahía Inglesa (Fig. 1) que han sido asignados preliminarmente al género Palaeospheniscus Moreno y Mercerat, 1891.

\section{MATERIALES Y MÉTODOS}

Los materiales aquí descritos se encuentran depositados en el Museo Nacional de Historia Natural (MNHN) de Santiago. Como materiales de comparación directa se consultaron los cráneos de las especies actuales y fósiles de las colecciones argentinas del Museo de La Plata (MLP), Museo de Ciencias Naturales "Bernardino Rivadavia" (MACN) de Buenos Aires y Museo Paleontológico Egidio Feruglio de Trelew (MEF).

Las descripciones de los materiales se efectuaron empleando la terminología de Baumel \& Witmer (1993) y las medidas, expresadas en milímetros, fueron tomadas con un Calibre Vernier.

La sistemática empleada sigue la propuesta de Acosta Hospitaleche (2004) y para el establecimiento de las edades se utilizó la Geología de la Carta de Caldera (Godoy et al. 2003).

\section{GEOLOGÍA DEL ÁREA}

La formación Bahía Inglesa fue definida por Rojo $^{5}$, indicando a Caldera (Fig. 1) como su localidad tipo y fue posteriormente enmendada por Marquardt ${ }^{6}$. Los sedimentos marinos que la componen son portadores de una amplia variedad de fósiles de vertebrados. Estas secuencias sedimentarias afloran en forma discontinua entre la franja costera de Caldera por el norte $\left(27^{\circ} 00^{\prime} S\right)$ y la desembocadura de la quebrada Totoral por el sur $\left(28^{\circ} 00^{\prime} S\right)$.

\footnotetext{
5 ROJO M (1985) Un aporte al conocimiento del Terciario marino: Formación Bahía Inglesa. Actas del Cuarto Congreso Geológico Chileno 1: 514-533.
} 


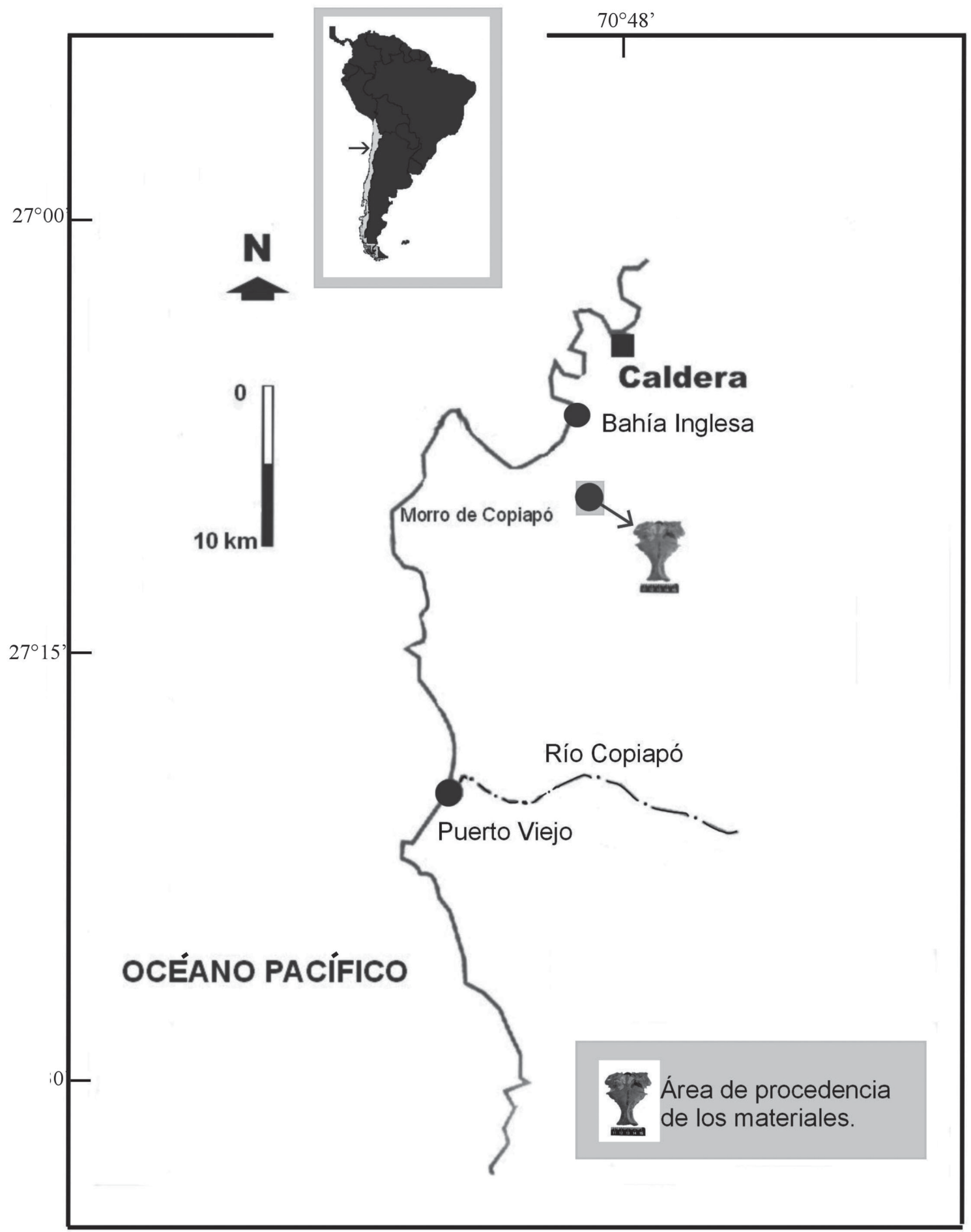

Fig. 1: Mapa de ubicación.

Location map. 
Predominan las litofacies de coquinas, areniscas y fangolitas, que incluyen diatomitas, fosforitas y cenizas retrabajadas. Las coquinas son de espesores cercanos a un metro, y generalmente están intercaladas con bancos de arena y guijarros. Su estructura es arenosa a conglomerádica, de color blanco amarillento y presentan material de Ostrea Sacco, 1897, Chlamys Röding, 1798 y Balanus da Costa, 1778. Los estratos de areniscas se presentan en bancos métricos masivos o con estratificación plana y cruzada en artesa. Pueden variar en una granulometría de fina a gruesa, así como de colores grises a pardo verdosas. La malacofauna es abundante, con frecuente bioturbación atribuida a poliquetos y bivalvos. También se registran contenidos variables de foraminíferos y abundantes restos de vertebrados (Long 1993, Walsh \& Hume 2001, Walsh \& Naish 2002).

Los materiales fósiles se han conservado en una matriz compuesta mayoritariamente por fosforita, la que se presenta en el sector de Bahía Inglesa, tanto en forma de depósitos mantiformes constituidos por capas decimétricas, así como depósitos asociados con sistemas de canales, los que son de distribución local (Rojo 1983).

La edad asignada a esta Formación, en base a estudios de paleomalacología, micropaleontología y relaciones estratigráficas, correspondería al Mioceno Medio a Plioceno Temprano, (Herm 1969, Marquardt 1999, Marquardt et al. 2000, Marchant et al. 20007, Godoy et al. 2003). Los registros de Globigerina bulloides (D’Orbigny 1826) y Neogloboquadrina pachyderma (Ehremberg, 1861) permiten asignar una edad máxima de Mioceno Medio, mientras que la presencia de Globigerina calida (Parker, 1962) y $G$. crassaformis (Galloway \& Wissler, 1927) permiten ubicar el techo de la secuencia en el Plioceno temprano. También una datación K-Ar

6 MARQUARDT C, N BLANCO, E GODOY, A LAVENU, L ORTLIEB, M MARCHANT \& N GUZMÁN (2000). Estratigrafía del Cenozoico superior en el área de Caldera $\left(26^{\circ} 45^{\prime}-28^{\circ} \mathrm{S}\right)$. Actas del Noveno Congreso Geológico Chileno 2: 588-592.

${ }^{7}$ MARCHANT M, C MARQUARDT, N BLANCO \& E GODOY (2000) Foraminíferos del área de Caldera $\left(26^{\circ} 45^{\prime}-28^{\circ} \mathrm{S}\right)$ y su utilización como indicadores cronoestratigráficos del Neógeno. Actas del Noveno Congreso Geológico Chileno, 5: 499-503. en biotita de una capa cinerítica ubicada hacia el techo de una de las sucesiones, en el sector de El Valle arrojó una edad de 7,6 \pm 1,3 Ma (Mioceno Tardío). Los restos fósiles proceden de estratos bajo esta línea de datación, por lo que son preliminarmente asignados a una edad comprendida entre Mioceno Medio a tardío (Godoy et al. 2003).

\section{PALEOAMBIENTE}

Los datos micropaleontológicos permiten interpretar esta unidad como una zona caracterizada por ambientes litorales a neríticos. Los foraminíferos béntonicos indican profundidades de hasta $150 \mathrm{~m}$ (Marchant 2000). De acuerdo a la microfauna se evidencia un predominio de periodos de aguas subantárticas, interrumpidos por eventos más cálidos en el Mioceno Medio y alrededor de los 6 Ma. Los depósitos de fosfatos y diatomitas señalan un ambiente de surgencia litoral de aguas profundas ricas en nutrientes (Marchant et al. 2000).

Las areniscas que se interdigitan con coquinas en la desembocadura del río Copiapó (al sur del área de recolección de los materiales), han sido interpretadas como pertenecientes a un delta proximal con influencia marina (Marchant et al. 2000).

\section{SISTEMÁTICA PALEONTOLÓGICA}

Orden Sphenisciformes Sharpe, 1891; Familia Spheniscidae Bonaparte, 1831; Subfamilia Palaeospheniscinae Simpson, 1946; Palaeospheniscinae cf. Palaeospheniscus Moreno y Mercerat, 1891.

Materiales: cráneos carentes de la región rostral: SGO-PV 1013 y SGO-PV 1063 colectados por uno de los autores (JC), SGOPV 1022 colectado por Martín Chávez, SGOPV 1035 colectado por Alejandro Marchini, SGO-PV 1036, colectado por Mario Suárez y SGO-PV 1054, colectado por Carlos Larraín.

Procedencia: sector central de la zona denominada Fosforita $\left(27^{\circ} 09^{\prime} 11^{\prime \prime} \mathrm{S}, 70^{\circ} 52^{\prime} 35^{\prime \prime}\right.$ O) de la localidad de Caldera, Región de Atacama, Chile. Formación Bahía Inglesa. Mioceno Medio-Tardío (Godoy et al 2003).

Descripción: su tamaño es apenas mayor al de S. magellanicus (Forster 1781) y mucho 
menor al de Spheniscus urbinai y $S$. megaramphus. (Tabla 1) Las cristae nuchalis temporalis son oblicuas y convergen hacia la crista nuchalis sagitalis como en Spheniscus, mientras que en Paraptenodytes Ameghino, 1891 son paralelas a la crista nuchalis transversa y en Pygoscelis Wagler, 1832, Aptenodytes Miller, 1778, Eudyptula Bonaparte, 1856 y Eudyptes Vieillot, 1816, no alcanzan la línea media. La crista nuchalis sagitalis presenta mayor extensión que en $S$. magellanicus y $S$. humboldti. Fossa temporalis más amplia que en Spheniscus pero de similar configuración, profundizándose caudalmente.

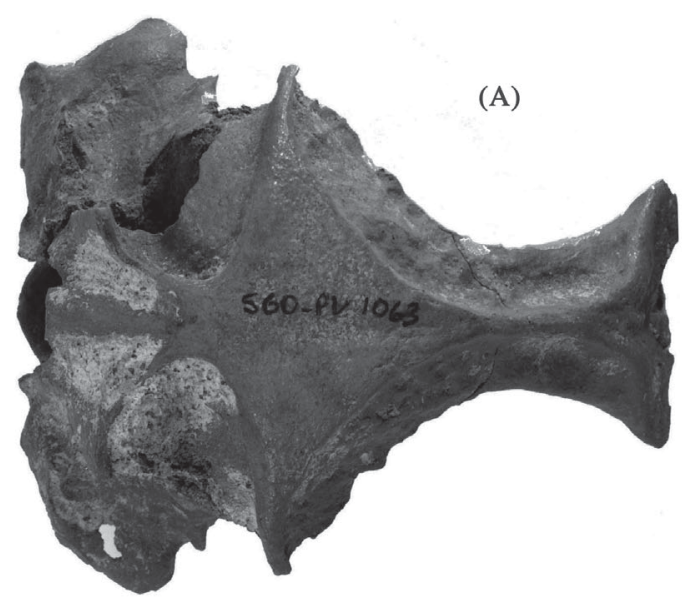

(B)

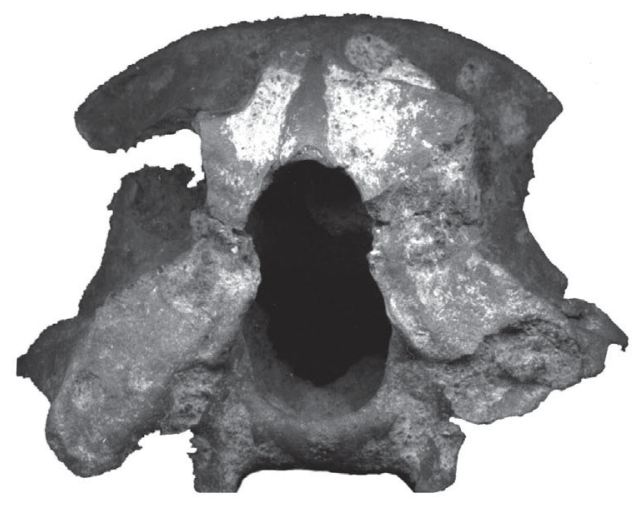

Fig. 2: Cráneo de Palaeospheniscus sp. SGOPV 1063, (A) vista dorsal, (B) vista occipital. Escala gráfica: $10 \mathrm{~mm}$.

Skull of Palaeospheniscus sp. SGO- PV 1063, (A) dorsal view, (B) occipital view. Scale bar: $10 \mathrm{~mm}$.
Fossae glandulae nassale más amplias que en Spheniscus y Eudyptula y menos separadas entre sí que en Paraptenodytes antarctica. La fosa se encuentra abierta lateralmente como en Paraptenodytes, Spheniscus, Eudyptula, Megadyptes Hombron y Jacquinot, $1841 \mathrm{y}$ Aptenodytes, mientras que en Pygoscelis y Eudyptes se desarrolla un reborde supraorbitario.

Frontales constituyendo una delgada cresta en forma de varilla en la región interorbitaria, como en Pygosceslis y Eudyptes y mucho menor que en los demás géneros comparados. Techo de la bóveda craneal de forma triangular en vista dorsal y proporcionalmente más pequeño que en las especies actuales, como ocurre en las especies fósiles de Spheniscus. Los processus paroccipitalis son amplios y se proyectan ventrocaudalmente. La región occipital presenta forma trapezoidal (en las especies actuales de Spheniscus es triangular).

\section{TABLA 1}

Medidas (expresadas en milímetros) de los ejemplares estudiados en este trabajo

Measurements (in milimeters) of the specimens studied in this contribution

\begin{tabular}{lccc}
\hline Medidas & $\begin{array}{c}\text { SGO- PV } \\
1063\end{array}$ & $\begin{array}{c}\text { SGO-PV } \\
1013\end{array}$ & $\begin{array}{c}\text { SGO- PV } \\
1036\end{array}$ \\
\hline Altura & 47,2 & - & 43 \\
Ancho postorbital & 55,8 & 58 & 60 \\
Ancho sulcus g. nasalis & 32 & - & - \\
Ancho región occipital & 55 & - & 56 \\
\hline
\end{tabular}

\section{DISCUSIÓN}

De confirmarse su asignación sistemática, estos restos constituirían los primeros cráneos conocidos para el género Palaeospheniscus. Los restos aquí estudiados son claramente distinguibles en su morfología de los géneros Paraptenodytes, Spheniscus y Pygoscelis, presentes en la misma unidad y para las cuales se conoce su esqueleto completo. Por otra parte, elementos apendiculares de Palaeospheniscus son abundantes en estos mismos sedimentos. No se ha hallado aún en esta unidad geológica ningún resto de Spheniscidae distinto de 
Palaeospheniscus, Paraptenodytes, Pygoscelis y Spheniscus a pesar del gran número de materiales del área descrita. La talla, robustez y caracteres generales observados en los materiales, se corresponden con aquellos esperables para este taxón.

Los cráneos aquí descritos constituyen un hallazgo de gran importancia debido a la escasez de restos craneanos que han sido dados a conocer hasta el momento en el registro paleontológico a nivel mundial y constituyen la primera cita de un resto craneal asignado a un Palaeospheniscinae.

La presencia conjunta en esta unidad de los diferentes géneros mencionados previamente sugiere por un lado una posible promediación temporal de los materiales (carácter no sincrónico de los depósitos), o por el otro, que los Spheniscinae y los Palaeospheniscinae habrían tenido una relación simpátrica en las costas chilenas entre el Mioceno Medio al Plioceno.

Dada la representación de especies mencionada en el registro paleontológico de elementos apendiculares y la elevadas abundancia de restos asignados a Palaeospheniscus, aunque posible, resulta sumamente improbable que estos restos pertenezcan a una especie diferente y aún no conocida.

Cabe señalar que este estudio contribuye a ampliar nuestro conocimiento sobre la anatomía esqueletaria de los Spheniscidae y constituye además un importante aporte al conocimiento de la avifauna fósil de Chile y en particular de la Formación Bahía Inglesa.

\section{AGRADECIMIENTOS}

Al investigador principal Daniel Frassinetti por permitirnos consultar la colección de Bahía Inglesa del MNHN, así como sus aportes al manuscrito. A José Yáñez por su revisión crítica al trabajo, a Sergio Hillebrandt por preparar los materiales, a Carlos Marquard por la información geológica proporcionada, y a la Empresa Minera Bifox Ltda. por permitirnos el ingreso a sus instalaciones. A Carlos Larraín y Patricio Covarrubias por su constante apoyo en terreno y a Herman Núñez por las fotografías. Este trabajo ha sido posible gracias al apoyo financiero del Consejo de las Américas a través del Proyecto "Estudio de Campo y Educación Ambiental Aplicada para la Conservación y Uso Sustentable del Patrimonio Paleontológico Costero de Bahía Inglesa-Caldera”.

\section{LITERATURA CITADA}

ACOSTA HOSPITALECHE C (2003) Paraptenodytes antarcticus (Aves: Sphenisciformes) en la formación Puerto Madryn (Mioceno Tardío temprano), provincia de Chubut, Argentina. Revista Española de Paleontología 18: 179-183.

ACOSTA HOSPITALECHE C (2004) Los pingüinos (Aves, Sphenisciformes) fósiles de Patagonia. Sistemática, biogeografía y evolución. Tesis doctoral, Facultad de Ciencias Naturales y Museo, Universidad Nacional de La Plata. La Plata, Argentina $321 \mathrm{pp}$.

ACOSTA HOSPITALECHE C \& M STUCCHI (2005) Nuevos restos terciarios de Spheniscidae (Aves, Sphenisciformes) procedentes de la costa del Perú Revista de la Sociedad Española de Paleontología 20: $1-5$.

BAUMEL J \& LM WITMER (1993) Osteología. En: Baumel J, A King, JE Breazile, H Evans \& JC Vanden Bergue (eds) Handbook of avian anatomy: Nomina Anatomica Avium: 45-132. Cambridge University Press, Cambridge, Massachusetts, USA.

FORDYCE R \& C JONES (1990) Penguin history and new fossil material from New Zealand. En: Davis LS \& JT Darby (eds) Penguin Biology: 419-446. Academic Press Inc., San Diego, California, USA.

GODOY E, C MARQUARDT \& N BLANCO (2003) Geología de la Carta Caldera. Región de Atacama. Carta Geológica de Chile, Serie Geología Básica escala 1:100.000. Servicio Nacional de Geología y Minería Chile.

HERM D (1969) Marines Pliözan und Pleistözan in Nord und Mittel Chile unter besonderen Berücksichtigung der Entwicklung der MolluskenFaunen. Zittelia (Germany) 2: 1-159.

LONG D (1993) Late Miocene and early Pliocene fish assemblages from the north central coast of Chile. Tertiary Research 14:117-126.

MARCHANT M (2000) Micropaleontología del área entre Caleta Obispito y Quebrada Los Burros, III Región. Servicio Nacional de Geología y Minería, Informe inédito. $52 \mathrm{pp}$.

MARQUARDT C (1999) Neotectónica de la franja costera y aportes a la geología regional entre Caldera y Caleta Pajonal $\left(27^{\circ}-27^{\circ} 45^{\prime}\right.$ S), III Región de Atacama, Chile. Tesis de Magíster, Departamento de Geología, Universidad de Chile. Santiago, Chile. 297 pp.

MARPLES B J (1952) Early Tertiary penguins of New Zeland. Geological Survey Paleontological Bulletin 20: 1-66.

MARPLES BJ (1960) A fossil penguin from the Late Tertiary of North Canterbury. Records of the Canterbury Museum (New Zealand) 7: 185-195.

ROJO M (1983) Geología y evaluación geológica del yacimiento de fosoforitas uraníferas de Bahía Inglesa, III Región. Inédito CORFO-CECHEN, Chile.

SIMPSON GG (1946) Fossil penguins. Bulletin of the American Museum of Natural History 87: 1-100. 
SIMPSON GG (1970) Miocene penguin from Victoria, Australia and Chubut, Argentina. Memoires of the Natural Museum of Victoria (Australia) 31: 17-24.

SIMPSON GG (1972) Conspectus of Patagonian fossil penguins. American Museum Novitates (USA) 2488: $1-37$.

STUCCHI M (2002) Una nueva especie de Spheniscus (Aves:Spheniscidae) de la Formación Pisco, Perú Boletín Geológico del Perú 94: 17-24

STUCCHI M, M URBINA \& A GIRALDO (2003) Una nueva especie de Spheniscidae del Mioceno Tardío de la Formación Pisco, Perú. Bulletin Institut Français d'Études Andines 32: 361-375

TAMBUSSI C, M REGUERO, S MARENSI \& $S$ SANTILLANA (en prensa) Crossvallia unienwillia, a new Spheniscidae (Sphenisciformes, Aves) from the Late Paleocene of Antarctica. Geobios.
TAMBUSSI C, C ACOSTA HOSPITALECHE, M REGUERO \& S MARENSI (en prensa) Late Eocene penguins from west Antarctica: systematic and biostratigraphy. En: Pirrie D, Francis J, \& A Crame (eds) Cretaceous Tertiary high-latitude palaeoenvironments, James Ross Basin, Antarctica. Geological Society of London, Special Publication.

TONNI EP (1980) The present state of knowledge of the Cenozoic birds of Argentina. Contributions in Science. Natural History Museum 330: 105-114.

WALSH SA \& JP HUME (2001) A new Neogene marine avian assemblage from north-central Chile. Journal of Vertebrate Paleontology 21: 484-491.

WALSH SA \& D NAISH (2002) Fossil seals from late neogene deposits in South America: a new pinniped (Carnivora, Mammalia) assemblage from Chile. Palaeontology 45: 821-842. 